\title{
Quantum chemical study of electron structure and charge transport properties of symmetric acenequinones
}

\author{
Denisa Cagardová, Martin Michalík, \\ Peter Poliak and Vladimír Lukeš \\ Department of Chemical Physics, Slovak University of Technology in Bratislava, \\ Radlinského 9, SK-812 37 Bratislava, Slovakia \\ martin.michalik@stuba.sk
}

\begin{abstract}
A systematic theoretical study using density functional theory is presented to estimate the structural, electronic, and charge-transfer characteristics of a symmetric fluorination of acenequinones outer rings. The change in aromaticity of model derivatives was described by different types of aromaticity indices. By considering a hopping mechanism and using the Marcus theory in combination with the Einstein-Smoluchowski relation, electronic drift mobilities were predicted for selected dimer configurations obtained from X-ray structures of anthraquinone, 6,13-pentacenequinone and its octafluorinated derivatives. The analysis of obtained data showed that the fluorination of the outer rings of acenequinones can lower the energy of the lowest unoccupied molecular orbital to the range from -3.0 to $-4.0 \mathrm{eV}$, i.e. typical for organic n-type semiconducting materials. Finally, potential electric semiconductivity of available X-ray structures relating to drift mobilities was discussed.
\end{abstract}

Keywords: flourination, semiconductors, aromaticity index, molecular orbital, aromatic structure

\section{Introduction}

Organic semiconductors based on a fused rings architecture are often used as materials for the construction of various optoelectronic devices. Over 400 organic materials showing a p-type behaviour (positive charges or holes as major carriers) have been reported (Wang et al., 2011). Some of them exhibit mobilities even higher than amorphous silicon $\left(\mu \approx 1.0 \mathrm{~cm}^{2} \mathrm{~V}^{-1} \mathrm{~s}^{-1}\right)$. The structurally simplest anthracene (Aleshin et al., 2004), tetracene (De Boer et al., 2003), pentacene (Klauk et al., 2002) and hexacene molecules have been successfully incorporated into organic field effect transistors (OFET). Measured hole mobility was improved as the number of fused rings was increased. Out of acenes, pentacene is a well-known commercial product exhibiting hole mobility $(\mu \approx 0.30$ to $3.00 \mathrm{~cm}^{2} \mathrm{~V}^{-1} \mathrm{~s}^{-1}$ ) which is dependent on the temperature when it is deposited in thin films (Klauk et al., 2003; Yin et al., 1997; Yakaphanoglu et al., 2012). The experiments performed on a very pure pentacene single crystal (Jurchescu et al., 2004) reported field effect hole mobility of $11.2 \mathrm{~cm}^{2} \mathrm{~V}^{-1} \mathrm{~s}^{-1}$. However, acenes, especially the longer ones, e.g. from heptacene to nonacene (Zade et al., 2010) are prone to oxidation mostly at their central ring. One solution is to block the oxidation centres with bulky alkyl substituents. Unfortunately, these substituents prevent co-facial stacking $\pi-\pi$ orientation, suppressing the attractive semiconductor properties (Shi et al., 2014).
Acenequinones are the products of oxidative degradation of acenes. The perturbation of $\pi$-delocalization over the quinoidal ring increases their chemical stability. Next, the planarity is maintained over the entire backbone and therefore acenequinones have a tendency for strong $\pi-\pi$ and hydrogen bond interactions in the solid state. On the other hand, the semiconducting properties of unsubstituted acenequinones are poor. For example, 6,13-pentacenequinone ( $\mathrm{Li}$ et al., 2010) is known as the major impurity in pentacene devices (Roberson et al., 2005) and it was successfully used as dielectric insulating layer for pentacene OFET devices (Jurchescu et al., 2007; Li et al., 2010).

Modern chemistry offers a wide range of possibilities to tune the electronic structure, such as Highest Occupied Molecular Orbital (HOMO) or Lowest Unoccupied Molecular Orbital (LUMO) energy levels of organic semiconducting materials (Brütting, 2005). Specifically, we can mention the introduction of strongly electronegative fluorine atoms to lower the LUMOs (Filo and Putala, 2010). The measurements of Liang et al. (Liang et al., 2010) showed that $\pi$-deficient fluorinated 6,13-pentacenequinones and corresponding N-heteropentacenequinones can work as chemically stable n-type organic semiconductors in thin film transistors with the electron mobility up to $0.1 \mathrm{~cm}^{2} \mathrm{~V}^{-1} \mathrm{~s}^{-1}$.

The Density Functional Theory (DFT) represents a suitable theoretical tool for orbital energy modelling of conjugated systems with a moderate computational cost (Hohenberg and Kohn, 1964; Runge 


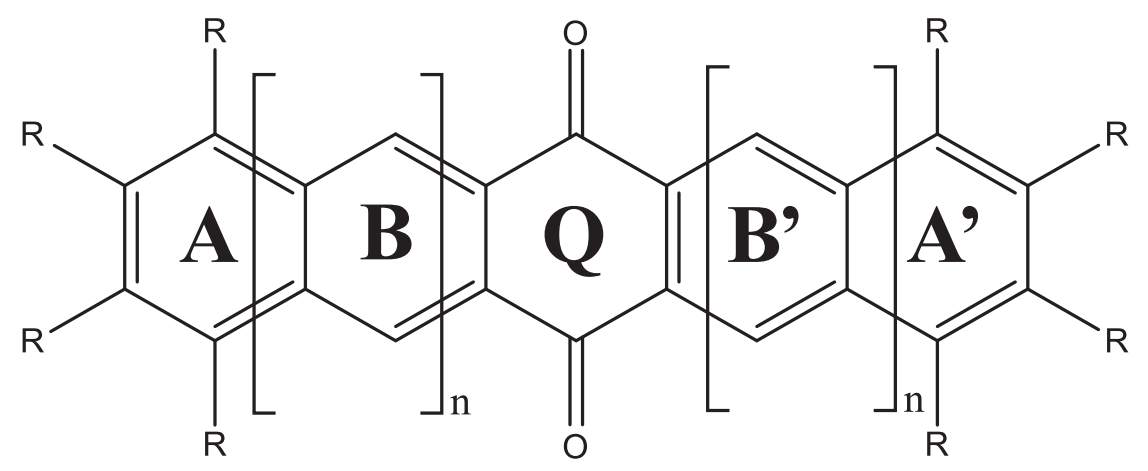

\begin{tabular}{ccc}
\hline Notation & $\mathrm{R}$ & $\mathrm{n}$ \\
\hline O3 & $\mathrm{H}$ & 0 \\
O5 & $\mathrm{H}$ & 1 \\
O7 & $\mathrm{H}$ & 2 \\
O9 & $\mathrm{H}$ & 3 \\
FO3 & $\mathrm{F}$ & 0 \\
FO5 & $\mathrm{F}$ & 1 \\
FO7 & $\mathrm{F}$ & 2 \\
FO9 & $\mathrm{F}$ & 3 \\
\hline
\end{tabular}

Fig. 1. Schematic structure and ring notation (capital letters) of studied molecules. Capital letters with single apostrophe indicate symmetry related rings.

and Gross, 1984). The hybrid DFT functional such as B3LYP (McCormick et al., 2013) is convenient for the gas-phase optimal geometry and electron structure investigation of molecules because it includes effects of electron correlation.

The main aim of this work is to present a systematic comparative study of the electronic structure of symmetric acenequinones and their octafluoro- derivatives (Fig. 1). The partial aims of this study are: (1) to calculate their optimal geometries in electroneutral, cationic and anionic charged states; (2) to evaluate the energies of frontier molecular orbitals and (3) to calculate the reorganisation energies and 4) to obtain Harmonic Oscillator Model of Electron Delocalization (HOMED) indices for studied molecules using the density functional theory. Next, for the selected compounds, the role of the chemical modification on the ideal $\pi$-stacking interaction energies will be analysed and the corresponding electric mobilities of dimers will be simulated. Finally, the evaluated quantities and trends will be compared with the available data from experiments.

\section{Theoretical}

\section{and computational methodology}

The quantum chemical calculations were performed using Gaussian 09 program package (Frisch et al., 2009). Geometries of all studied molecules in electroneutral and charged states were calculated using DFT method with hybrid B3LYP (Becke's three parameter Lee-Yang-Parr) functional (Becke, 1988; Lee et al., 1988). For all calculations, the $6-311+\mathrm{G}(2 \mathrm{~d}, \mathrm{p})$ basis set was used for all atoms (Rassolov et al., 1998; Hariharan and Pople, 1973). Optimized structures were confirmed to be real minima of adiabatic potential surfaces by vibrational analysis (no imaginary frequencies).

The model dimer structures were studied by means of B3LYP functional including the Grimme's GD3BJ dispersion corrections on van der Waals in- teractions (Grimme et al., 2010). The B3LYP energy of each monomer was corrected on Basis Set Superposition Error (BSSE) (Boys and Bernardi, 1970) using the Counterpoise method (Simon et al., 1996; Xantheas, 1996). The molecules and molecular orbitals were visualised using the Molekel program package (Flukiger et al., 2002).

The characterization of aromaticity is usually done by using a set of indices based on physical properties (Cyrañski et al., 2002). As a structure-based analysis, we discuss the geometrical changes of $\mathrm{C}-\mathrm{C}$ aromatic bond lengths in selected acenequinones using HOMED. These changes are relative to the reference bond lengths obtained from the optimized geometries of ethane, ethene and benzene using B3LYP functional in combination with 6-311+G(2d,p) basis set (Rassolov et al., 1998; Hariharan and Pople, 1973). In case of X-ray structures, the Harmonic Oscillator Model of Heterocyclic Electron Delocalization (HOMHED) (Frizzo and Martins, 2012) was used for aromaticity description. Reference bond lengths considered in calculations of HOMHED and the normalization factor $\alpha$ were obtained from X-ray diffraction measurements. Both of these aromaticity indices can be expressed by the equation

$$
\begin{gathered}
\operatorname{HOM}(\mathrm{H}) \mathrm{ED}= \\
=1-\frac{1}{n^{\prime}}\left\{\alpha_{\mathrm{CC}} \sum_{\mathrm{i}=1}^{n^{\prime}}\left(R(\mathrm{CC})_{\mathrm{opt}}-R(\mathrm{CC})_{\mathrm{i}}\right)^{2}\right\}
\end{gathered}
$$

where $n$ 'represents a number of bonds considered in the investigated aromatic ring and $R(\mathrm{CC})_{\mathrm{i}}$ is the real $\mathrm{CG}$ bond length. In the case of HOMED indices, the optimal B3LYP/6-311+G(2d,p) benzene bond length $1.3919 \AA$ was taken as the reference $R_{\text {opt }}$. In the case of HOMHED, the proposed reference CC optimal bond length $\left(R_{\text {opt }}\right)$ is of $1.387 \AA$ (Frizzo and Martins, 2012). Normalization factor $\alpha$ used in relation for HOMED is of $86.3 \AA^{-2}$ (Lukeš at al., 2018) and for HOMHED index determination equals $78.5 \AA^{-2}$ (Frizzo and Martins, 2012). 
The ionization potentials (IPs) as energy required to abstract electron from the molecule were calculated from an energy difference between a cationic and a neutral state. Similarly, electron affinities (EAs) present energy related to electron addition to the molecule. The EAs were obtained as energy difference between anionic and neutral state. Vertical ionization potentials (VIPs) and electron affinities (VEAs) represent change to the charged state without a geometry relaxation. Adiabatic quantities include geometry relaxation of charged species.

Based on the idea of a hopping mechanism, the charge-transport (CT) rate between two adjacent molecular moieties is given by Marcus formula (Marcus, 1993; Sakanoue et al., 1999; Malagoli and Brédas, 2000)

$$
k=t^{2}\left(\frac{\pi}{h^{2} k_{\mathrm{B}} \lambda^{ \pm} T}\right)^{1 / 2} \exp \left(-\frac{\lambda^{ \pm}}{4 k_{\mathrm{B}} T}\right)
$$

where $t$ represents an intermolecular charge transfer integral between interacting molecular moieties, $h$ is the Planck constant, $k_{\mathrm{B}}$ is the Boltzmann constant, $T$ is the thermodynamical temperature and $\lambda$ represents the reorganization energy of a hole $\left(\lambda^{+}\right)$or an electron $\left(\lambda^{-}\right)$. In practice, the calculations of $t$ integrals are simplified by considering the geometry of the electroneutral dimer. Application of Koopmans' theorem (KT) (Koopmans, 1934) is another major simplification relying on the one-electron approximation. Using this approximation, the absolute value of the charge transfer integral between two moieties is given by the relation

$$
t=\frac{\varepsilon_{\mathrm{LUMO}+1[\mathrm{HOMO}]}-\varepsilon_{\mathrm{LUMO}[\mathrm{HOMO}-1]}}{2}
$$

where $\varepsilon$ are the energies of corresponding molecular orbitals taken from the closed-shell configuration of the electroneutral geometry of a dimer. Because of its simplicity, the KT in combination with the Energy-Splitting-in-Dimer (ESD) (Lemaur et al., 2004) approach is currently the most frequently used method for the estimation of transfer integrals in organic semiconductors.

Another important necessity is to consider the geometry change caused by the transition from an electroneutral to a charged state of the molecule. The reorganization energy is usually described as the sum of internal and external contributions. The external reorganization energy is neglected. The internal reorganization energy refers to the energy required for the geometry relaxation upon the change from a neutral state to a specified charged molecular state and vice versa. This energy (Marcus, 1993; Brédas et al., 2004;
Wang et al., 2014) is obtained from the adiabatic potential energy surface and it is given as

$$
\begin{gathered}
\lambda^{ \pm}=\lambda_{1}^{ \pm}+\lambda_{2}^{ \pm}= \\
=\left[E_{ \pm}\left(Q_{N}\right)-E_{ \pm}\left(Q_{ \pm}\right)\right]+\left[E_{N}\left(Q_{ \pm}\right)-E_{N}\left(Q_{N}\right)\right]
\end{gathered}
$$

where $E_{ \pm}\left(Q_{N}\right)$ is the total energy of the charged state in the neutral geometry, $E_{ \pm}\left(Q_{ \pm}\right)$is the total energy of the charged state in the charged state geometry, $E_{N}\left(Q_{+}\right)$is the total energy of the neutral state in the charged state geometry, and $E_{\mathrm{N}}\left(Q_{\mathrm{N}}\right)$ is the total energy of the neutral state in the neutral geometry (Brédas et al., 2004; Wang et al., 2014).

Under constant temperature, the electric drift mobility $\mu$ can be calculated from the EinsteinSmoluchowski relation (Maier and Ankerhold, 2010; Cornil et al., 2002)

$$
\mu=\frac{e k d^{2}}{2 n k_{\mathrm{B}} T}
$$

where $e$ is the elementary charge, $d$ is the centroidto-centroid distance for electron hopping between two moieties in the dimer and $k$ is the charge transfer hopping rate. The symbol $n$ denotes space dimensionality and equals 3 in this case.

\section{Results and Discussion}

\subsection{Aromaticity, geometrical structure and reorganization energy}

The B3LYP optimal geometries of all studied molecules except FO3 in electroneutral and monocharged states are planar. Therefore, the aromaticity will be affected by bond length changes within the planar structures. The comparison of aromaticity indices is shown in Fig. 2. The electroneutral anthraquinone molecule (O3) exhibits the largest $\operatorname{HOMED}\left(\mathrm{A} / \mathrm{A}^{\prime}\right)$ value of 0.9967 . On the other hand, HOMED of the central quinone ring $Q$ with four $(1.4904 \AA)$ and another two equivalent bond lengths $(1.4034 \AA)$ of 0.4377 indicates poor aromaticity. With the increasing number of condensed benzene rings, a decrease of $\operatorname{HOMED}\left(\mathrm{A} / \mathrm{A}^{\prime}\right)$ is observed from 0.9967 for $\mathbf{O} 3$ to 0.8679 for the $\mathbf{O 9}$ molecule (see Fig. 2a). As shown in Fig. 2b, the same trend is followed by the $\operatorname{HOMED}(\mathrm{Q})$ index of the central quinone ring, i.e. a decrease from the value of 0.4377 to 0.3996 . Thus, central rings of both non- and fluorinated acenequinones possess non-aromatic character. Interestingly, aromaticity indices for rings $\mathrm{B} / \mathrm{B}^{\prime}$ in $\mathbf{O} 5, \mathbf{O} 7$ and $\mathbf{O 9}$ molecules (see Fig. 1 for notation) are slightly larger (ranging from 0.9448 for $\mathbf{O 5}$ to 0.9029 for $\mathbf{0 9}$ ) than the $\operatorname{HOMED}\left(\mathrm{A} / \mathrm{A}^{\prime}\right)$ ones. In comparison with acenequinones, fluorination of outer rings of $\mathbf{O 5}, \mathbf{O} 7$ and O9 molecules causes an increase of HOMED 
indices for condensed benzene rings $\mathrm{A} / \mathrm{A}^{\prime}, \mathrm{B} / \mathrm{B}$ ', $\mathrm{C} / \mathrm{C}$, $\mathrm{D}$ and results in a decrease of $\operatorname{HOMED}(\mathrm{Q})$ parameters in fluorinated analogues. In the case of the FO3 molecule, the $\operatorname{HOMED}\left(\mathrm{A} / \mathrm{A}^{\prime}\right)$ index decreases by 0.003 with respect to $\mathbf{O 3}$ as the fluorinated molecule is no more planar (see Fig. 2a).

The cumulative $\mathrm{HOM}(\mathrm{H}) \mathrm{ED}^{*}$ value is expressed as an average value of all $\mathrm{HOM}(\mathrm{H}) \mathrm{ED}$ indices included in a molecule

$$
\operatorname{HOM}(\mathrm{H}) \mathrm{ED}^{*}=\frac{\sum_{i=1}^{N} \operatorname{HOM}(\mathrm{H}) \operatorname{ED}(\mathrm{i})}{N}
$$

where $i$ is an identification of the ring and $N$ is the number of rings in the molecule. The smallest cumulative HOMED* index was found for the FO3 molecule $(0.7890)$. It is caused by the non-planarity of this molecule due to the fluorine substitution. With the exception of FO3, the fluorination causes slight HOMED* increase by about 0.02 (see Fig. 2c). For the sake of comparison, HOMHED parameters for anthraquinone, pentacenequinone and their octafluoro- derivatives are also illustrated.

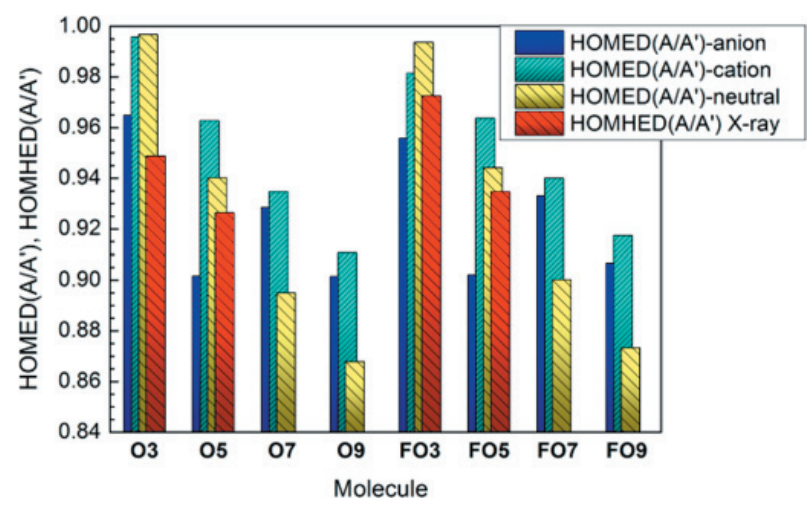

a)
Unlike X-ray crystallography, quantum chemical calculations help us to estimate how the molecular geometry and electronic structure are changed upon the electrochemical oxidation or reduction. The energy difference reflecting geometrical changes upon charging is called a reorganization energy. The B3LYP/6-311+G(2d,p) calculated values for both charged states are summarized in Table 1. Reorganization energies of both charge carriers in fluorinated molecules are higher than reorganization energies of their non-fluorinated analogues. The B3LYP reorganization energy of the octafluoro-anthraquinone (FO3) molecule connected with the cationic state formation $\left(\lambda^{+}\right)$ is of $267 \mathrm{meV}$ and it is the highest value of the hole reorganization energy among all molecules under study. Within the non-fluorinated and fluorinated acenequinone group, there is a significant decrease of reorganization energy with increasing number of condensed units. In case of the non-fluorinated compounds, their $\lambda^{+}$ values change from $62 \mathrm{meV}$ for $\mathbf{0 9}$ to $133 \mathrm{meV}$ for the $\mathbf{0 3}$ molecule. Substituting by fluorine

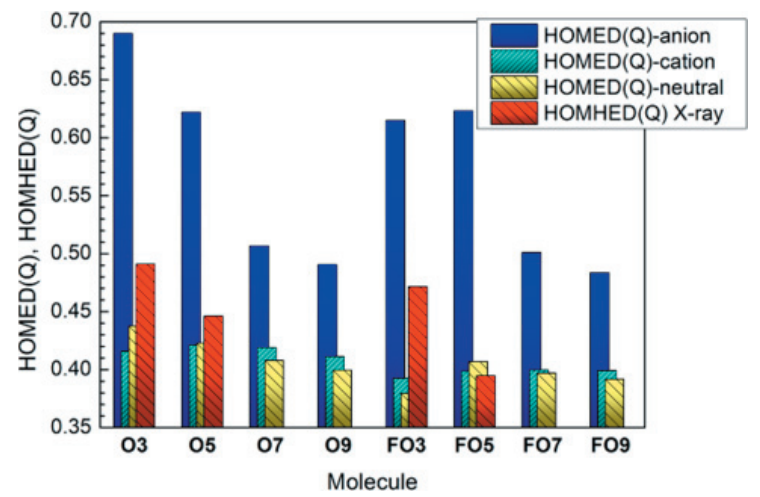

b)

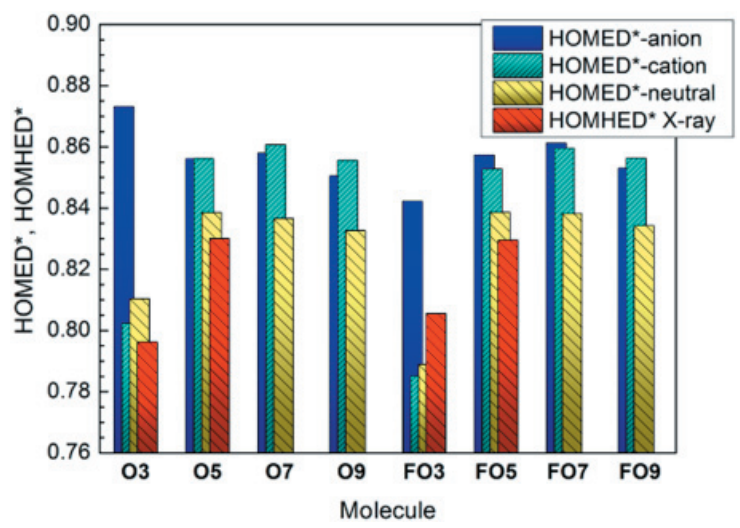

c)

Fig. 2 Aromaticity indices HOMED and HOMHED for a) A/A' rings, b) central quinone (Q) rings and $\mathbf{c}$ ) averaged cumulative B3LYP HOMED* and HOMHED* parameters of studied molecules in neutral, cationic and anionic states. HOMHED indices are obtained from X-ray structures of O3, FO3, FO5 and 05 molecules. 
atoms has high tendency to enhance the values of reorganization energies. Electron reorganization energies $\left(\lambda^{-}\right)$for the fluorinated acenequinone derivatives range from $91 \mathrm{meV}$ for FO9 to $445 \mathrm{meV}$ for FO3, while $\lambda^{-}$for non-fluorinated ones varies from $66 \mathrm{meV}$ for $\mathbf{O 9}$ to $356 \mathrm{meV}$ for $\mathbf{O 3}$.

Tab. 1. B3LYP vertical ionisation potentials (VIP), adiabatic ionisation potentials (AIP), vertical electron affinities (VEA), adiabatic electron affinities (AEA), reorganization energies for hole and electron transfer $\left(\lambda^{+}\right.$ and $\lambda^{-}$, respectively) of studied molecules. All energies are in $\mathrm{eV}$.

\begin{tabular}{lcccccc}
\hline Molecule & VIP & AIP & VEA & AEA & $\lambda^{+}$ & $\lambda^{-}$ \\
\hline O3 & 9.05 & 9.00 & 1.56 & 1.74 & 0.133 & 0.356 \\
O5 & 7.84 & 7.79 & 1.49 & 1.62 & 0.103 & 0.278 \\
O7 & 6.99 & 6.95 & 1.85 & 1.90 & 0.077 & 0.083 \\
O9 & 6.42 & 6.39 & 2.20 & 2.23 & 0.062 & 0.066 \\
FO3 & 9.61 & 9.48 & 2.29 & 2.52 & 0.267 & 0.445 \\
FO5 & 8.41 & 8.30 & 2.18 & 2.35 & 0.208 & 0.329 \\
FO7 & 7.54 & 7.47 & 2.42 & 2.48 & 0.135 & 0.120 \\
FO9 & 6.92 & 6.87 & 2.66 & 2.71 & 0.097 & 0.091 \\
\hline
\end{tabular}

\subsection{Frontier molecular orbitals}

The energy levels of frontier molecular orbitals (FMOs) are key parameters for predicting the carrier injection ability and stability of the material. High HOMO and low LUMO energy levels ensure efficient hole and electron injections ( $\mathrm{Lv}$ et al.,
2012). Based on the optimized structures of the neutral state, energy levels have been calculated and are shown in Fig. 3. The B3LYP HOMO energy of studied acenequinones ranges from $-7.36 \mathrm{eV}$ for $\mathbf{O} 3$ to $-5.46 \mathrm{eV}$ for $\mathbf{0 9}$. The energy level of HOMO for pentacenequinone is of $-6.55 \mathrm{eV}$ which is in quite good agreement with the experimental value of $-6.21 \mathrm{eV}$ (Liang et al., 2010). Fluorination of outer rings in acenequinones leads to the decrease of HOMO and LUMO energies. The range of shifts varies from $0.66 \mathrm{eV}$ for $\mathbf{O 3} /$ FO3 to $0.50 \mathrm{eV}$ for fluorination of $\mathbf{0 9}$. In the case of FO5, the calculated HOMO energy is of $-7.13 \mathrm{eV}$. The experimental HOMO energy obtained from the LUMO energy level and the optical gap is of -6.32 eV (Liang et al., 2010). A fluorine substitution may ensure the optimal setting of a LUMO energy with respect to n-type semiconductors. The calculated LUMO energies vary from $-2.81 \mathrm{eV}$ (O5) to $-3.88 \mathrm{eV}$ (FO3). Within the acenequinone model molecules, we indicated a slight decrease of LUMO energy levels from $-2.81 \mathrm{eV}$ for $\mathbf{O 5}$ to $-3.17 \mathrm{eV}$ for the $\mathbf{O 9}$ molecule (see Fig. 3). The $\mathbf{O 3}$ molecule is excluded from this trend. The same effect is observable within fluorinated derivatives. LUMO energies vary from the initial value of $-3.52 \mathrm{eV}$ for FO5 to $-3.64 \mathrm{eV}$ for FO9. The reliability of the calculated B3LYP energies of frontier molecular orbitals can be estimated from a comparison with experimental data obtained from spectroelectrochemical measurements. The experimental value of the LUMO energy of the FO5 molecule

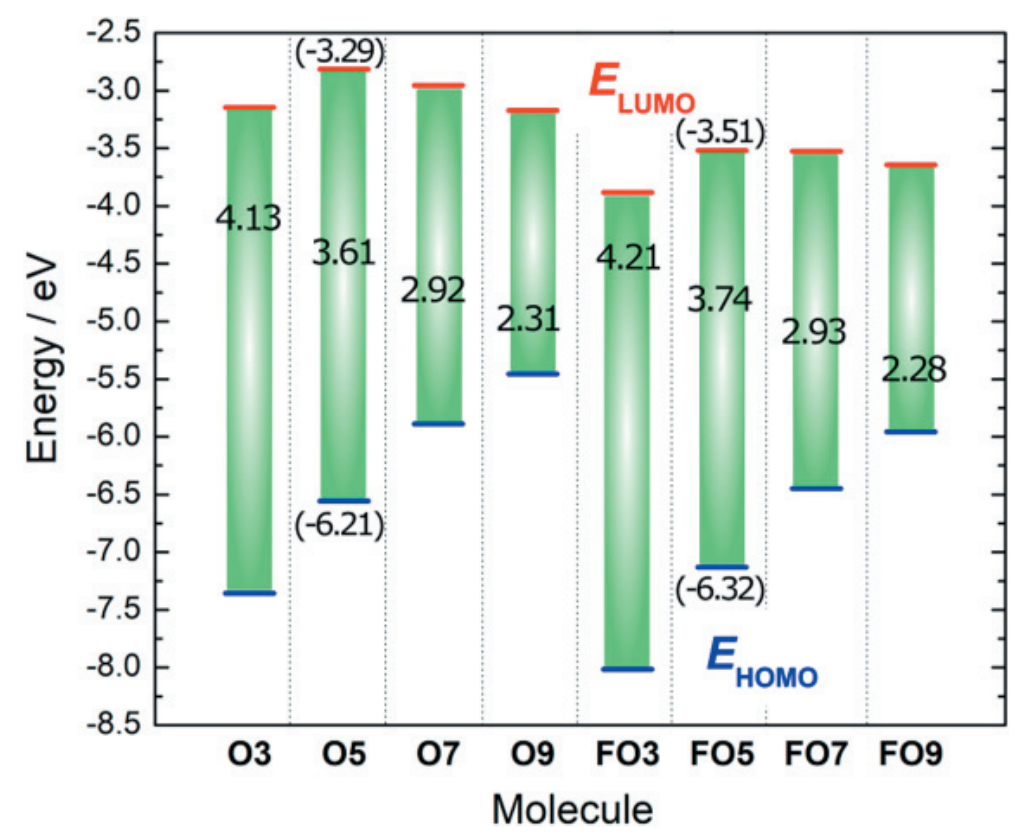

Fig. 3. Energy diagram of B3LYP frontier molecular orbitals for neutral states of studied molecules. Green bands and corresponding numbers indicate energy gaps $\Delta E_{\mathrm{g}}$. Experimental values (in eV) are in parentheses (Liang et al., 2010). 
obtained from the half-wave reduction potential vs. ferrocenium/ferrocene by Liang et al. is of $-3.51 \mathrm{eV}$ (Liang et al., 2010), which is in an excellent agreement with the calculated one $(-3.52 \mathrm{eV})$. These values are also indicated in parentheses of Fig. 3 . The maximal value of LUMO energy level was observed for the FO3 molecule $(-3.88 \mathrm{eV})$. However, the increase of HOMO energies is more significant than the decrease of LUMO ones resulting in more pronounced effect of HOMO-LUMO energy gap $\left(\Delta E_{g}\right)$. It represents another important physical factor for the electric semiconductor property. For the acenequinones, $\Delta E_{g}$ values are changed from 2.31 to $4.13 \mathrm{eV}$ and for their fluorinated analogues they are in a range from 2.28 to $4.21 \mathrm{eV}$. The maximal value of $\Delta E_{g}$ was determined for the non-planar FO3 molecule. The mutual comparison of obtained data illustrates how the elongation of condensed units in a molecule can decrease the $\Delta E_{g}$ values.

As depicted in Fig. 4, the HOMO and LUMO orbitals of acenequinone derivatives are distributed over the entire molecule and reveal the typical $\pi$-type molecular orbital character that demonstrates their good carrier transport property. Shapes of

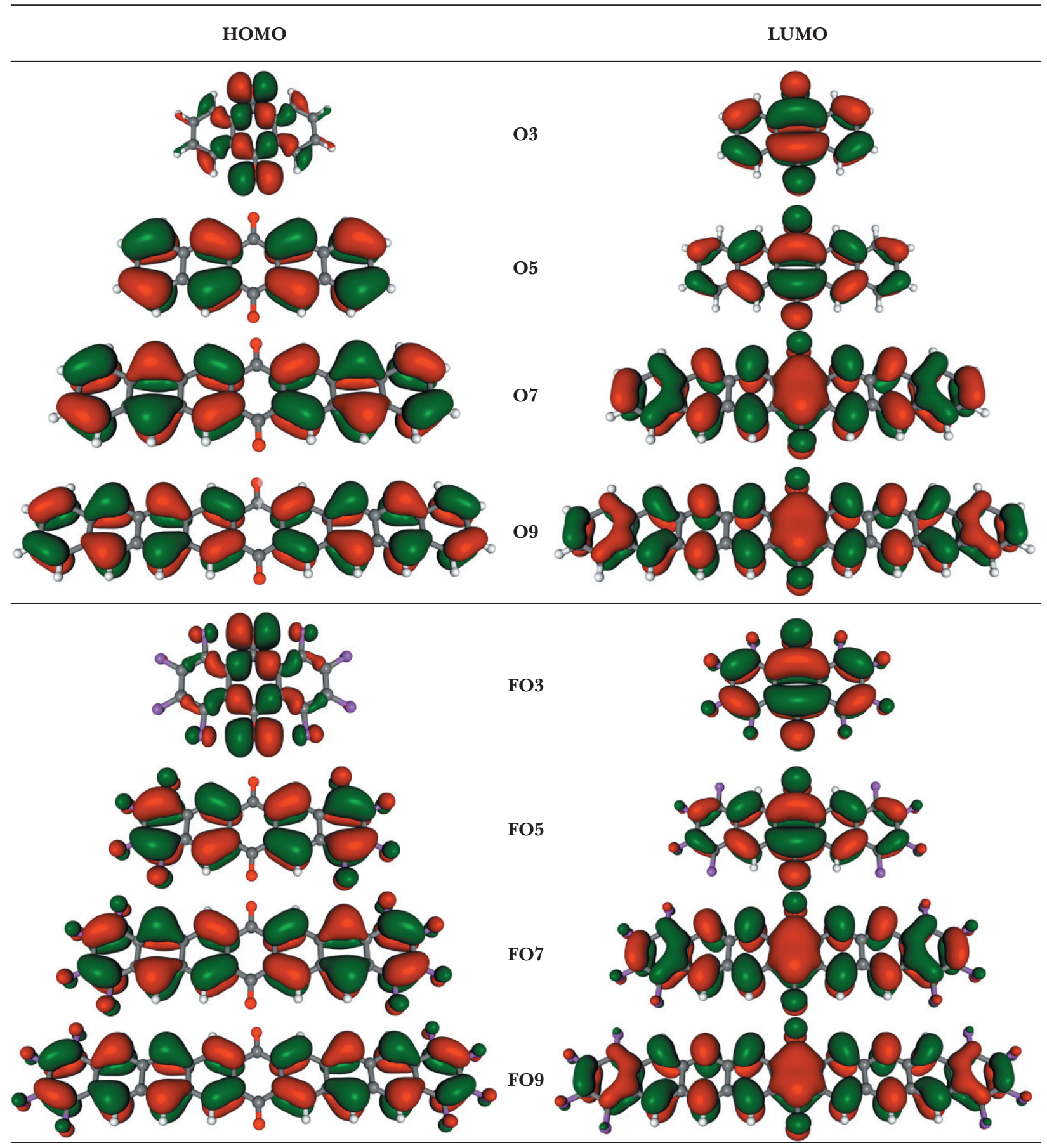

Fig. 4. Shapes of B3LYP HOMO and LUMO orbitals for studied molecules (0.025 a.u. isosurface). 
HOMO orbitals show no significant change in relation to the molecule elongation. One exception is the anthraquinone molecule (and its fluorinated derivative FO3), which possess slightly different shapes of HOMO. In contrast to HOMO, the lobes of LUMO are delocalized over the fluorine atoms as well as over the bonds parallel with the smaller axis direction. In all studied molecules, the $\pi$-electron delocalization of LUMOs over the central quinone ring $(Q)$ is present. Within the set of model acenequinone molecules and their fluorinated derivatives, significant changes of frontier MOs shapes are not observable.

\subsection{Ionization potentials and electron affinity}

The electrochemical properties of investigated organic molecules are proportionally related to their ionization energy (IP) and electron affinity (EA). Both the theoretical LUMO energy and EA express the energy associated with electron addition to a molecule. The frontier molecular orbital approach is routinely used in the interpretation of spectroelectrochemical measurements in solution. On the other hand, the electron affinity and ionization potential are more rigorous. They are commonly used in the interpretation of electrical solid-state experiments (Cagardová et al., 2019). The calculated gas-phase B3LYP vertical ionization potentials (VIPs) and vertical electron affinities (VEAs) are collected in Table 1. In the case of the octafluoroanthraquinone model molecule, the VIP value is of $9.61 \mathrm{eV}$ which represents the largest value of VIP among all the studied molecules. Vertical ionization potential of the $\mathbf{O} 3$ molecule is of $9.05 \mathrm{eV}$ and it decreases with the increasing number of condensed benzene units within the non-fluorinated group of model molecules. On the other hand, the molecule elongation causes an increase of vertical electron affinities (VEAs) within the model series from $1.56 \mathrm{eV}$ for $\mathbf{O 3}$ to $2.20 \mathrm{eV}$ for $\mathbf{0 9}$.

Within the model structures of acenequinones, adiabatic IPs and EAs range from $6.39 \mathrm{eV}$ (O9) to $9.00 \mathrm{eV}(\mathbf{O 3})$ and from $1.74 \mathrm{eV}(\mathbf{O 3})$ to $2.23 \mathrm{eV}$ (O9), respectively. According to previous studies, the adiabatic IPs of selected air-stable p-type materials range from $5.680 \mathrm{eV}$ to $6.786 \mathrm{eV}$, while the adiabatic EA for the n-type ones should be less than $4.0 \mathrm{eV}$. The air-stable n-type ones should be over $2.80 \mathrm{eV}$ (Liu et al., 2010; Chang et al., 2010).

\subsection{Charge carrier mobility simulations for ideal $\pi$-stacking dimers}

Stacking of aromatic rings in solid state represents the second key factor which affects the macroscopic electric properties of organic molecules. Geometries of interacting monomers in various planar and transversal dimer configurations were obtained from available experimental X-ray measurements of O3 (Chen et al., 2015), O5 (Liang et al., 2010), FO3 (Chetkina and Popova, 1973) and FO5 (Liang et al., 2010) molecules (see Fig. 5). Interaction energies were calculated for X-ray geometries without optimization using the B3LYPGD3BJ functional, which is able to model weak attraction forces between different molecules.

Theoretical investigations by Brédas and co-workers (Brédas et al., 2002) have shown that displacements along the long and the short axis of $\pi$-stacked semiconductor molecules strongly affect the intermolecular electronic couplings, in such a way that closely depends on the phase and nodal properties of the frontier molecular orbitals. Another interesting findings on the molecular packing are the four weak hydrogen bonds linking the neighboring $\pi$-stacks of $\pi$-deficient pentacenequinones. As shown in Fig. 6a, hydrogen $\mathrm{O} \cdots \mathrm{H}$ bonds are present between moieties in the planar dimer configuration of anthraquinone denoted as $\mathbf{0 3}$. Distances between hydrogen atoms and the strongly electronegative oxygen ones equal $3.12 \AA$. The molecular packing of fluorinated pentacenequinone FO5 in the X-ray structure (Fig. 6b) features $\pi-\pi$ stacking as well and weak hydrogen bonds between hydrogen and fluorine/oxygen atoms. Moieties in crystal structure form infinite one-dimensional stacks with a $\pi-\pi$ interplanar distance of $3.34 \AA$, which is slightly shorter than the interplanar distance in graphite (3.35 A) (Lian et al., 2010). As illustrated in Fig. 6b (dashed lines), the short distances between the neighboring stacks are the four weak $\mathrm{C}-\mathbf{H} \cdots \mathbf{O} / \mathbf{F}$ hydrogen bonds with a self-complementary ADAD-DADA pattern ( $\mathrm{D}$ is hydrogen donor, and $\mathrm{A}$ is hydrogen acceptor). These hydrogen bonds play an important role in the structure stability. In these four weak hydrogen bonds, the estimated $\mathrm{H} \cdots \mathrm{F}$ and $\mathrm{H} \cdots \mathrm{O}$ distances are almost the same ( 4.10 and $4.62 \AA$, respectively). In case of crystal structure of pentacenequinone O5, the molecular packing features weak $\mathrm{C}-\mathbf{H} \cdots \mathbf{O}$ hydrogen bonds. Distances within hydrogen bonds are ranging from 2.42 $\AA$ for the planar P5 dimer configuration to $7.01 \AA$ for the $\mathrm{P} 6$ dimer configuration (see Fig. 6c-f). To conclude, Fig. 6 shows that it is plausible to suppose that the presence of hydrogen bonds will significantly affect the real molecular arrangement in solid state.

Depending on the molecular type and dimer configuration, the interaction energies range from -2.05 to $-73.76 \mathrm{~kJ} \mathrm{~mol}^{-1}$ (see Table 2 ). The smallest centroid-to-centroid distance is of $4.69 \AA$ with the corresponding interaction energy of $-73.76 \mathrm{~kJ} \mathrm{~mol}^{-1}$ in the planar (P1) dimer configuration of the 


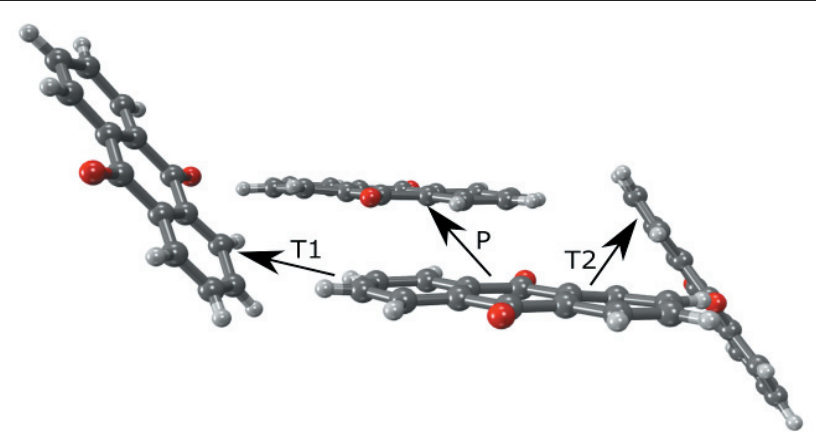

a)

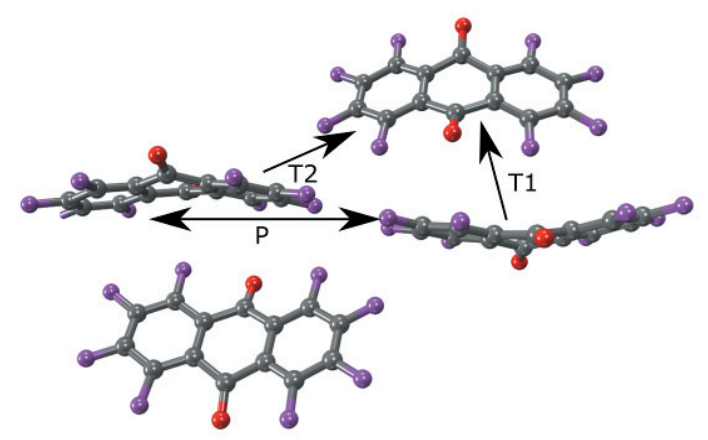

b)

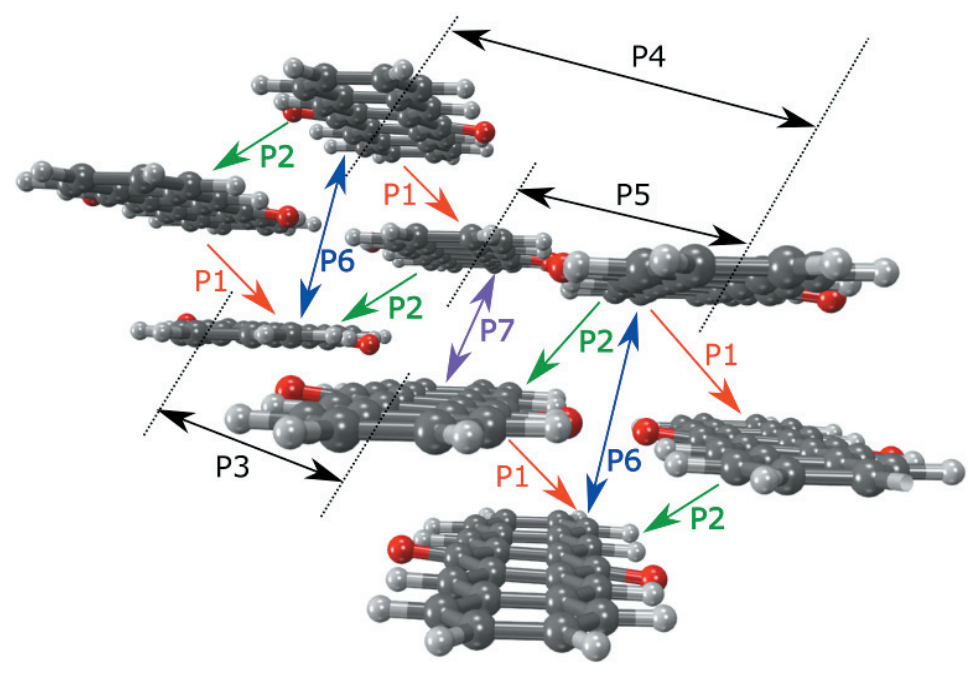

c)

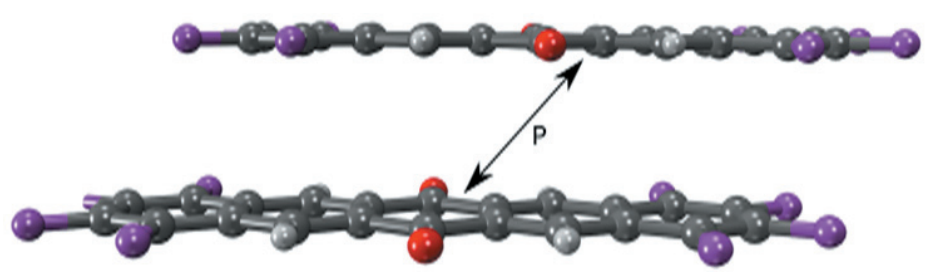

d)

Fig. 5. Molecular packing diagrams and dimer type neighbors in the X-ray structure of a) O3 (Chen et al., 2015); b) FO3 (Chetkina et al., 1973); c) O5 (Liang et. al., 2010) and d) FO5 molecules (Liang et. al., 2010). 

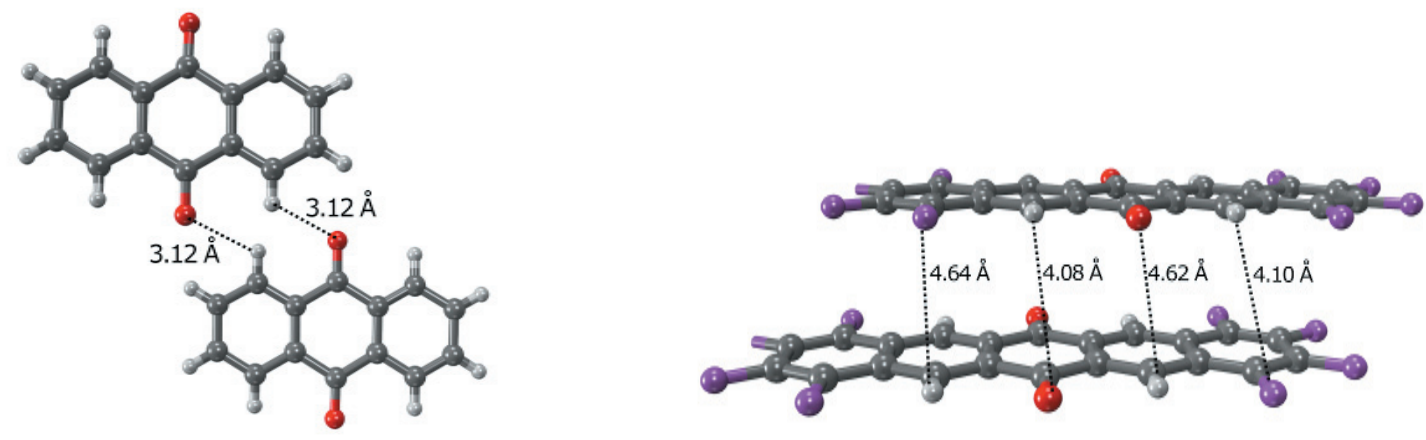

P-dimer

O3

a)

P-dimer FO5

b)
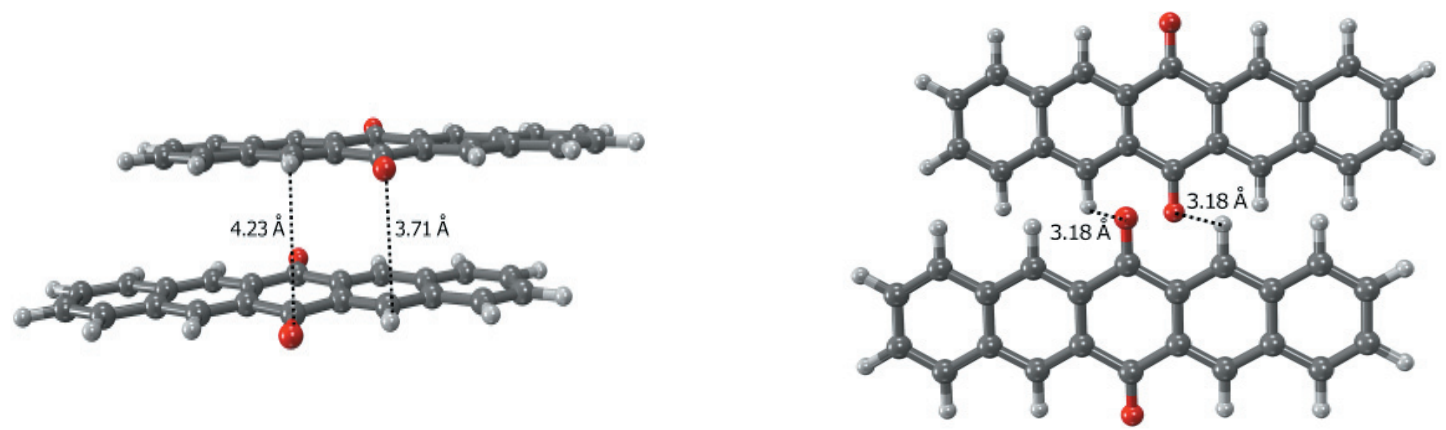

P1-dimer O5

c)

P2-dimer O5

d)

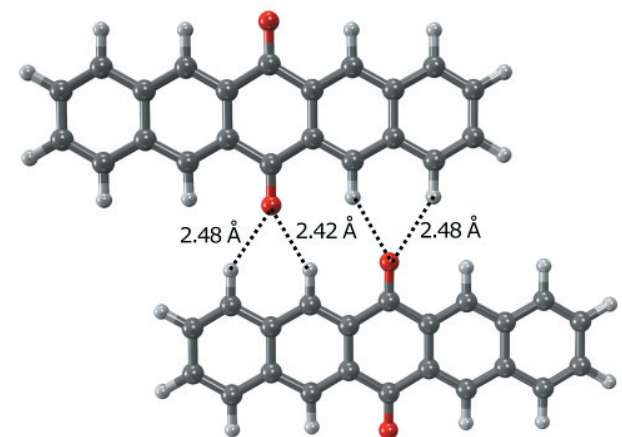

P5-dimer O5

e)

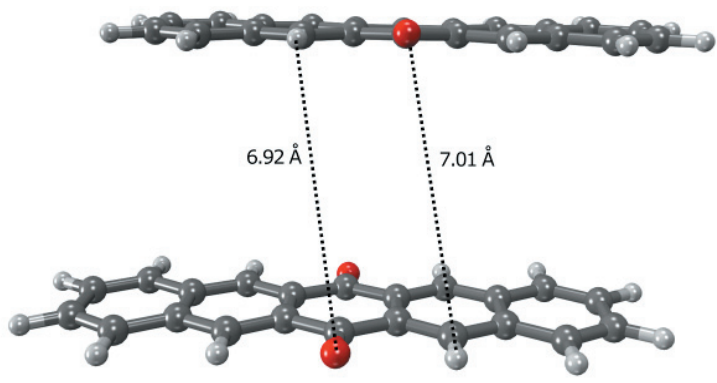

P6-dimer O5

f)

Fig. 6. Hydrogen bonds in a) P-dimer configuration within the X-ray structure of $\mathbf{O 3}$ (Chen et al., 2015);

b) P-dimer configuration obtained from the X-ray of FO5 (Liang et. Al., 2010); and

c) planar P1, d) P2, e) P5 and

f) P6 dimer configuration obtained from the X-ray structure of $\mathbf{O 5}$ (Liang et. Al., 2010).

Numerical data stand for hydrogen bond lengths.

O5 molecule. Considering the hopping mechanism and using the Marcus theory, drift mobilities of hole and electron transfer found for this dimer configuration of $\mathbf{O 5}$ are of 1.07 and $0.15 \mathrm{~cm}^{2} \mathrm{~V}^{-1} \mathrm{~s}^{-1}$, respectively. On the other hand, slightly larger hole drift mobility of $1.22 \mathrm{~cm}^{2} \mathrm{~V}^{-1} \mathrm{~s}^{-1}$ was found for the P2 configuration. The other five dimers have negligible low mobilities. Therefore, in average, macroscopic isolating property of 05 was confirmed. Analogously, X-ray structures of $\mathbf{O 3}$ and 
Tab. 2. B3LYP-GD3BJ equilibrium centroid-to-centroid distances $(d)$ in $\AA$, interaction energies $\left(\Delta E_{\text {int }}\right)$ in $\mathrm{kJ} \mathrm{mol}^{-1}$ and hole/electron mobilities $\left(\mu_{\mathrm{h}} / \mu_{\mathrm{e}}\right)$ in $\mathrm{cm}^{2} \mathrm{~V}^{-1} \mathrm{~s}^{-1}$ of selected studied dimer configurations obtained from O3, FO3, O5 and FO5 X-ray structures.

\begin{tabular}{lrrrrlrrrc}
\hline Dimer & \multicolumn{1}{c}{$\boldsymbol{d}$} & \multicolumn{1}{c}{$\Delta \boldsymbol{E}_{\text {int }}$} & $\mu_{\mathrm{h}}$ & $\mu_{\mathrm{e}}$ & Dimer & \multicolumn{1}{c}{$\boldsymbol{d}$} & $\Delta \boldsymbol{E}_{\text {int }}$ & $\mu_{\mathbf{h}}$ & $\mu_{\mathrm{e}}$ \\
\hline O3(P) & 7.85 & -17.78 & 0.04 & 0.02 & FO3(P) & 12.43 & -2.09 & 0.00 & 0.00 \\
O3(T1) & 10.01 & -6.49 & 0.87 & 0.07 & FO3(T1) & 5.62 & -30.33 & 0.20 & 0.37 \\
O3(T2) & 8.07 & -16.99 & 2.01 & 0.28 & FO3(T2) & 10.76 & -6.77 & 0.26 & 0.04 \\
\hline O5(P1) & 4.69 & -73.76 & 1.07 & 0.15 & FO5(P) & 4.89 & -75.19 & 0.02 & 0.17 \\
O5(P2) & 5.99 & -38.41 & 1.22 & 0.07 & & & & \\
O5(P3) & 13.79 & -5.98 & 0.00 & 0.00 & & & & & \\
O5(P4) & 13.45 & -2.05 & 0.00 & 0.00 & & & & & \\
O5(P5) & 8.16 & -31.80 & 0.01 & 0.03 & & & & & \\
O5(P6) & 7.02 & -4.73 & 0.00 & 0.00 & & & & & \\
O5(P7) & 14.17 & -7.78 & 0.11 & 0.02 & & & & & \\
\hline
\end{tabular}

its fluorinated derivative FO3 was used to provide the analysis of contributions to the macroscopic mobility. In contrast to the pentacenequinone molecule, mostly transversal dimer configurations contribute to the macroscopic drift mobility (see Table 2). As illustrated in Fig. 5b, molecular structure of octafluoro-anthraquinone contains strongly electronegative fluorine and oxygen atoms resulting in repulsive interactions between the moieties in the X-ray structure. Moreover, also the small interaction energy of $-2.09 \mathrm{~kJ} \mathrm{~mol}^{-1}$ (Table 2) for planar configuration (P) shows destabilization and non-planarity of the system. On the other hand, the interaction energies of $\mathbf{O 3}$ are slightly higher. As shown, planar configuration $(\mathrm{P})$ provides the large interaction energy $\Delta E_{\text {int }}=-17.78 \mathrm{~kJ} \mathrm{~mol}^{-1}$ due to the stabilization of the dimer by hydrogen $\mathrm{O} \cdots \mathrm{H}$ bonds (see Fig. 6a).

\section{Conclusions}

The model series of selected acenequinones and their octafluoro-derivatives were investigated. Fluorination of outer rings in the smallest anthraquinone molecule causes a structure deformation resulting in a non-planarity of FO3. The evaluated energies of frontier molecular orbitals and the electronic band gaps are mutually compared together with the adiabatic electronic intramolecular reorganization energies. The lowest LUMO energy $(-3.88 \mathrm{eV})$ was evaluated for FO3 indicating its n-type semiconducting properties. On the other hand, the best model structure providing p-type semiconductivity is $\mathbf{0 9}$ due to its highest LUMO energy $(-3.17 \mathrm{eV})$. The analysis of frontier molecular orbital shapes shows that HOMOs of $\mathbf{O 3}$ and FO3 differ from the remaining studied molecules. In case of LUMO, differences are observable in the shapes of $\mathbf{O 3}$ and $\mathbf{O 5}$. The effect of fluorination on the shapes of FMOs is minimal.

Finally, X-ray structures of O3, O5, FO3 and FO5 were examined from the point of view of intermolecular hydrogen bond stability and the corresponding electric drift mobilities were simulated. Anthraquinone can be considered as a p-type semiconductor because of its relatively high hole mobility $\left(2.01 \mathrm{~cm}^{2} \mathrm{~V}^{-1} \mathrm{~s}^{-1}\right)$ in the transversal T2 dimer configuration. On the contrary, the transversal T1 dimer configuration in octafluoro-anthraquinone FO3 provides the largest contribution to the macroscopic drift mobility $\left(0.37 \mathrm{~cm}^{2} \mathrm{~V}^{-1} \mathrm{~s}^{-1}\right)$. The transversal dimer configurations in the $\mathrm{X}$-ray structure of octafluoro-anthraquinone contribute to macroscopic drift mobility significantly, thus the distortion can be neglected. Although pentacenequinone is considered to be an electric insulator, the analysis of its electric mobility contributions shows that planar P1 and P2 dimer configurations have relatively high hole mobilities. However, from the statistical point of view, there are five more planar configurations with negligible mobilities resulting in the noteworthy decrease of its macroscopic mobility. On the other hand, the octafluoropentacenequinone provides only one planar dimer configuration in the crystal with predicted electron mobility of $0.17 \mathrm{~cm}^{2} \mathrm{~V}^{-1} \mathrm{~s}^{-1}$ at room temperature which is in good agreement with experimental value.

\section{Acknowledgment}

The work has been supported by Slovak Grant Agency (1/0601/15) and Slovak Research and Development Agency (APVV-15-0079). The authors would like to thank for financial contribution from the STU Grant scheme for Support of Young Researchers. We are grateful to the HPC center at the Slovak University of Technology 
in Bratislava, which is a part of the Slovak Infrastructure of High Performance Computing (SIVVP project, ITMS code 26230120002, funded by the European region development funds, ERDF) for the computational time and resources made available.

\section{References}

Aleshin AN, Lee JY, Chu SW, Kim JS, Park WY (2004) Appl Phys Lett 84: 5383.

Becke AD (1988) Phys Rev A 38: 3098-3100

Boys SF, Bernardi F (1970) Mol Phys 19: 553-566.

Brédas JL, Beljonne D, Coropceanu V, Cornil J (2004) Chem Rev 104: 4971-5003.

Brédas JL, Calbert JP, da Silva Filho DA, Cornil J (2002) Proc Natl Acad Sci USA. 99: 5804-5809.

Brütting W (2005) Organic Semiconductors, University of Augsburg, Germany.

Cagardová D, Michalík M, Poliak P, Lukeš V (2019) J Mol Struc 1175: 297-306.

Cornil J, Lemaur V, Calbert JP, Brédas JL (2002) Adv Mater 14: 726.

Cyrañski MK, Krygowski TM, Katritzky AR, Schleyer PvR (2002) J Org Chem 67: 1333-1338.

De Boer RWI, Klapwijk TM, Morpurgo AF (2003) Appl Phys Lett 83: 4345.

Filo J, Putala M (2010) J Electr Eng 61: 314-320.

Flukiger P, Luthi HP, Sortmann S, Weber J (2002) Molekel 4.3, Swiss National Supercomputing Centre, Manno, Switzerland.

Frizzo CP, Martins MAP (2012) Struct Chem 23: 375-380.

Frisch MJ, Trucks GW, Schlegel HB, Scuseria GE, Robb MA, Cheeseman JR, Scalmani G, Barone V, Mennucci B, Petersson GA, Nakatsuji H, Caricato M, Li X, Hratchian HP, Izmaylov AF, Bloino J, Zheng G, Sonnenberg JL, Hada M, Ehara M, Toyota K, Fukuda R, Hasegawa J, Ishida M, Nakajima T, Honda Y, Kitao O, Nakai H, Vreven T, Montgomery JA Jr., Peralta JE, Ogliaro F, Bearpark M, Heyd JJ, Brothers E, Kudin KN, Staroverov VN, Keith T, Kobayashi R, Normand J, Raghavachari K, Rendell A, Burant JC, Iyengar SS, Tomasi J, Cossi M, Rega N, Millam JM, Klene M, Knox JE, Cross JB, Bakken V, Adamo C, Jaramillo J, Gomperts R, Stratmann RE, Yazyev O, Austin AJ, Cammi R, Pomelli C, Ochterski JW, Martin RL, Morokuma K, Zakrzewski VG, Voth GA, Salvador P, Dannenberg JJ, Dapprich S, Daniels AD, Farkas O, Foresman JB, Ortiz JV, Cioslowski J and Fox DJ (2009) Gaussian 09, Revision D.01, Gaussian, Inc. Wallingford CT.

Grimme S, Antony J, Ehrlich S, Krieg H (2010) J Chem Phys 132: 154104 .

Hariharan PC, Pople JA (1973) Theor Chim Acta 28: 213-222.

Hohenberg P, Kohn W (1964) Inhomogeneous electron gas, Phys Rev 136: B864-B871.

Chang YC, Kuo MY, Lu HF, Chao I (2010) J Phys Chem C 114: 11595 .

Chen YJ, Yang SC, Tsai CC, Chang KC, Chuang WH, Chu WL, Kovalev V, Chung WS (2015) Chem Asian J 10(4).
Chetkina LA, Popova EG (1973) Kristallografiya 18: $1162-1168$

Jurchescu OD, Baas J, Palstra TTM (2004) Appl Phys Lett 84: 3061-3063.

Jurchescu OD, Popinciuc M, van Wees BJ, Palstra TTM (2007) Adv Mater 19: 688.

Klauk H, Halik M, Zschieschang U, Schmid G, Radlik W, Weber W (2002) J Appl Phys 92: 5259.

Koopmans T (1934) Physica 1: 104-113.

Lee C, Yang W, Parr RG (1988) Phys Rev B 37: 785-789.

Lemaur V, Da Silva Filho DA, Coropceanu V, Lehmann M, Geerts Y, Piris J, Debije MG, Van De Craats AM, Senthilkumar K, Siebbeles LDA, Warman JM, Brédas JL, Cornil J (2004) J Am Chem Soc 126: 3271-3279.

Li Z, Du J, Tang Q, Wang F, Xu J, Yu JC, Miao Q (2010) Adv Mater 22: 3242-3246.

Liang Z, Tang Q, Liu J, Li J, Yan F, Miao Q (2010) Chem Mater 22: 6438-6443.

Lin YY, Gundlach DJ, Nelson SF, Jackson TN (1997) IEEE Electron Device Lett. 18: 606-608.

Liu CG, Mao SW, Kuo MY (2010) J Phys Chem C 114: 22316.

Lukeš V, Cagardová D, Michalík M, Poliak P (2018) Synth Metls 240: 67-76.

Lv A, Puniredd SR, Zhang J, Li Z, Zhu H, Jiang W, Dong H, He Y, Jiang L, Yan L, Pisula W, Meng Q, Hu W, Wang Z (2012) Adv Mater 24: 2626-2630.

Maier SA, Ankerhold J (2010) Phys Rev E 81: 021107.

Malagoli M, Brédas JL (2000) Chem Phys Lett 327: $13-17$.

Marcus RA (1993) Rev Mod Phys 65: 599-610.

McCormick TM, Bridges CR, Carrera EI, DiCarmine PM, Gibson GL, Hollinger J, Kozycz LM, Seferos DS (2013) Macromolecules, 46: 3879-3886.

Ośmiałowski B, Raczyńska ED and Krygowski TM (2006) J Org Chem 71(10): 3727-3736.

Rassolov VA, Pople JA, Ratner MA, Windus TL (1998) J Chem Phys 109: 1223-1229.

Roberson LB, Kowalik J, Tolbert LM, Kloc C, Zeis R, Chi X, Fleming R, Wilkins CJ (2005) Am Chem Soc 127: 3069-3075.

Runge E, Gross EKU (1984) Phys Rev Lett 52: 997-1000. Sakanoue K, Motoda M, Sugimoto M, Sakaki S (1999) J Phys Chem A 103: 5551-5556.

Shi ZF, Black HT, Dadvand A, Perepichka DF (2014) J Org Chem 79: 5858-5860.

Simon S, Duran M, Dannenberg JJ (1996) J Chem Phys 105: 11024-11031.

Wang C, Dong H, Hu W, Liu Y, Zhu D (2011) Chem Rev 112: 2208-2267.

Wang L, Li P, Xu B, Zhang H, Tian W (2014) Org Electron 15: 2476-2485.

Xantheas SS (1996) J Chem Phys 104: 8821-8824.

Xiao J, Duong HM, Liu Y, Shi W, Ji L, Li G, Li S, Liu XW, Ma J, Wudl F, Zhang Q (2012) Angew Chem Int Ed 51: 6094

Yakuphanoglu F, Mansouri S, Bourguiga R (2012) Synt Met 162: 918-923.

Zade SS, Bendikov M (2010) Angew Chem Int Ed 49: 4012. 